\title{
Perioperative Events in Living and Deceased Donor Liver Transplant Recipients: A Case Control Study
}

\author{
Bhargavi Gali*,, David J. Plevak ${ }^{1}$, David M. Nagorney ${ }^{2}$, Charles B. Rosen², Julie K. Heimbach², \\ Ross A. Dierkhising ${ }^{3}$ and James Y. Findlay ${ }^{1}$
}

${ }^{I}$ Department of Anesthesiology, Mayo Clinic, 200 First Street SW, Rochester, MN, USA

${ }^{2}$ Department of Surgery, Mayo Clinic, Rochester, MN, USA

${ }^{3}$ Division of Biostatistics, Mayo Clinic, Rochester, MN, USA

\begin{abstract}
Living donor liver transplantation (LD) has been implemented as an alternative to deceased donor transplantation (DD). We reviewed the perioperative course of recipients of LD and recipients of DD at our institution with specific aims to compare intraoperative events, early complication rates, resource utilization, and patient survival. Methods: Following Institutional Review Board (IRB) approval, the first forty LD cases were retrospectively matched by age, sex, and primary reason for transplant with controls that underwent DD between June 30, 2000 and January 25, 2005. Preoperative data, intraoperative variables, and immediate postoperative data were collected. Statistical analysis included signed rank test, McNemar's test, Wald statistics, and stratified Cox proportional hazards model. Results: Calculated Model for End Stage Liver Disease (MELD) scores were higher for DD (median 18 vs. 14 with p=0.04). Anesthesia time was longer in LD (median 7.1 vs. 6.5, $\mathrm{p}=0.02$ ). Hospital length of stay (LOS) was higher in LD (median $12 \mathrm{vs.} 8$ days, $\mathrm{P}=0.002)$. Seven of the $40(17 \%) \mathrm{LD}$ were deceased at the time of data collection, as were four $(10 \%)$ of the DD. Conclusions: Comparison of DD and LD at our institution revealed few significant differences in perioperative variables. LD may have more postoperative complications and longer hospital stays but similar patient survival.
\end{abstract}

Keywords: Transfusion requirements, perioperative morbidity, biliary complications, wound infections, intraoperative complications.

\section{INTRODUCTION}

Living donor liver transplantation (LD) has been implemented as an alternative to deceased donor liver transplantation (DD). With approximately 17,000 candidates awaiting $\mathrm{DD}$, living donor transplantation offers an alternative for patients unlikely to receive a deceased donor liver. Over 300 living donor liver transplants were performed each year from 2002 to 2005 in the United States [1]. Anesthesiologists, surgeons, and intensivists have had much experience with $\mathrm{DD}$, but much less in caring for recipients of living donor liver transplants. As living donation becomes an accepted alternative to deceased donation, the safety of this option is a concern. Several publications have reported complications and outcomes associated with LD [2-5], however direct comparisons with DD recipients may be confounded by differences in the patient characteristics of those undergoing $\mathrm{LD}$ versus $\mathrm{DD}[6]$.

Adult to adult LD was first performed at our institution in 2000; by 2005 we had carried out 40 cases. We reviewed these cases for intraoperative, early postoperative events, and outcomes. These were compared with a matched group of DD cases. We hypothesized that with case matching the

*Address correspondence to this author at the Department of Anesthesiology, Mayo Clinic, 200 First Street SW, Rochester, MN, USA; Tel: 507-284-9695; Fax: 50-284-0120; E-mail: gali.bhargavi@mayo.edu complication frequency and outcomes would be similar in both groups.

\section{PATIENTS AND METHODS}

This study was performed with approval of the institutional review board of Mayo Clinic. Data were abstracted from prospectively maintained transplantation and anesthesia databases as well as by review of patient medical records. Each LD case was matched by age (within 12 years), gender, and etiology of liver disease leading to transplantation, to a DD primary liver transplant case. All transplants occurred during the 2000-2005 time period.

Preoperative investigations and screening for both $\mathrm{LD}$ and DD patients were similar. All patients, DD and LD, met UNOS minimum listing criteria. The decision to list for transplantation was made by a multidisciplinary group including transplant surgeons; transplant hepatologists; specialists in anesthesia, critical care, and infectious diseases; nurse coordinators; social workers; and other allied health members of our transplant team.

The first $40 \mathrm{LD}$ procedures at the Mayo Clinic were performed by two experienced liver transplant staff surgeons (CBR and JKH). All transplants were performed with caval sparing hepatectomy without use of venous bypass. Donor liver grafts were perfused with Viaspan (UW solution) and prepared for implantation on a back table. Deceased donor iliac veins were used as interposition grafts for recipients 
with hilar cholangiocarcinoma [7] and occasionally for reconstruction of large segment $\mathrm{V}$ or segment VIII veins. Right liver grafts were implanted with a right hepatic vein to recipient right hepatic vein anastomosis, and larger inferior hepatic and caudate veins were sewn directly to the recipient cava with an end-to-side anastomosis during 22 of the operations. The donor right hepatic vein was sewn to the recipient left/middle hepatic vein for a recipient with complete situs inversus. Segment V and/or segment VII veins were reconstructed in 5 patients using either deceased donor iliac veins or the recipient middle hepatic vein which had been preserved during the hepatectomy. The donor right portal vein was sewn to the recipient portal vein, and deceased donor iliac veins were used as interposition grafts for all 9 cholangiocarcinoma (CCA) patients. Following portal reperfusion, arterial reconstruction was performed between the donor right or replaced right hepatic artery and the recipient right, left, proper, or common hepatic artery. Biliary reconstruction was performed with either a duct-toduct anastomosis or a Roux Y hepatojejunostomy over an internal biliary stent.

The deceased donor transplant procedures were performed by a staff surgeon and transplant surgery fellow (either as primary surgeon). All patients underwent caval-sparing hepatectomy without use of venous bypass. Implantation was accomplished with an end-to-end anastomosis between the donor suprahepatic vena cava and the recipient left/middle hepatic venous trunk; and end-to-end portal venous anastomosis; and arterial reconstruction. A deceased donor iliac artery graft to the infrarenal aorta was used for all hilar cholangiocarcinoma patients and patients with inadequate common hepatic arterial flow. Biliary reconstruction was either with a duct-to-duct anastomosis over a biliary tube inserted through the donor cystic duct stump or a Roux Y choledochojejunostomy over a biliary tube brought out through the bowel wall.

Intraoperative anesthetic management for all transplants was carried out by members of the liver transplant anesthesia group. Anesthetic management for both groups was the same, consisting of general anesthesia using isoflurane with fentanyl supplementation. Routine invasive monitoring was utilized, including direct arterial pressure and pulmonary artery catheterization. Rapid infusion and cell saver devices were used for all cases.

Postoperatively all patients were transferred to the transplant ICU where the same immediate postoperative management protocol, including the use of a ventilator weaning protocol, was followed.

\section{Data Collection and Analysis}

Database and patient records were reviewed for anesthesia time, surgical time, blood product transfusion, and significant pulmonary or hemodynamic events (Table 1). Postoperative information collected included blood product transfusion, cardiac and pulmonary complications, biliary complications, infections, length of intubation, acute renal dysfunction, portal vein or hepatic artery thrombosis (HAT), and hospital length of stay (LOS).

\section{Table 1. Data Collection}

Intraoperative cardiopulmonary events:
Mean arterial pressure less than 50\% baseline
CPR
Hypoxemia requiring intervention (documented on anesthetic record)
Arrhythmia requiring intervention
Postoperative Hospitalization
Occurrence of "Postoperative event"
Prolonged intubation (>48hrs)
Reintubation
At least one episode of MAP less than 50\% baseline
Institution of CPR
Other significant alteration in cardiorespiratory status requiring
emergent intervention (specify)
Occurrence of "Postoperative diagnosis"

Myocardial infarction- defined as diagnosis by treating physician, or Troponin $>0.1$

Deep venous thrombosis- defined as diagnosis by physician, or positive LE Doppler study

Pulmonary

Atelectasis requiring respiratory therapy involvement

Pneumonia defined as diagnosis by treating physician, or new infiltrate on CXR requiring antibiotic therapy

Pulmonary embolus- defined as diagnosis by treating physician, or positive $\mathrm{CT} /$ angio

Wound infection

Ileus

Incisional hernia

Cholestasis

Nerve Palsy

Bleeding requiring transfusion (donor), or $>5$ units PRBCs (recipient)

Acute renal failure (requiring dialysis)

Cerebral vascular accident-defined as diagnosis by physician, or new area of infarction or hemorrhage on CT

Sepsis- evidence of infection plus a systemic response as manifested by an elevated temperature, tachycardia, increased respirations, leukocytosis or an impaired peripheral leukocyte response

Biliary tract

Leak

Biloma

Stenosis

Portal vein thrombosis

Liver failure or persistent cholestasis, defined as total bili> 5 after postop day 10

Recipients Only

Hepatic artery thrombosis (HAT)

Graft nonfunction

Hepatic encephalopathy

Posthospitalization

Mortality (at time of data collection)

Readmission for any of above complications within 3 months of transplantation

Definitions for complications are given in Table $\mathbf{1}$. Complications for a period of 3 months after transplantation were included, as was readmission to the hospital within 3 
Table 2. Patient Demographics

\begin{tabular}{|l|c|c|}
\hline \multicolumn{1}{|c|}{ Characteristic } & Living Donor (n=40) & Deceased Donor $(\mathbf{n}=\mathbf{4 0})$ \\
\hline \hline Age, mean \pm SD, $y$ & $51 \pm 11$ & $50 \pm 13$ \\
\hline Sex: & $27(67.5 \%)$ & $25(62.5 \%)$ \\
\hline Male & $13(32.5 \%)$ & $15(37.5 \%)$ \\
\hline Female & $1(2.5 \%)$ & $2(5 \%)$ \\
\hline Body Mass Index $\left(\mathrm{kg} / \mathrm{m}^{2}\right):(\mathrm{p}=0.02):$ & $24(60 \%)$ & $10(25 \%)$ \\
\hline$<19$ & $11(27.5 \%)$ & $17(42.5 \%)$ \\
\hline $19-25$ & $4(10 \%)$ & $11(27.5 \%)$ \\
\hline$>25-30$ & $14(14)$ & $17(18)$ \\
\hline$>30$ & & $17(42.5 \%)$ \\
\hline MELD, mean (median) $(\mathrm{p}=0.04)$ & $9(22.5 \%)$ \\
\hline Primary reason for transplant: & $3(7.5 \%)$ \\
\hline Cholestatic liver disease & $3(7.5 \%)$ \\
\hline Alcohol-related hepatitis & $8(20 \%)$ \\
\hline Hepatitis C $(1$ with HCC also) & \\
\hline Other (includes AlH, HCC, NASH, cryptogenic, and neuroendocrine) & \\
\hline
\end{tabular}

Key:

MELD model for end stage liver disease

PBC primary biliary cirrhosis

PSC primary sclerosing cholangitis

$\dagger \quad 4$ with diagnosis of PSC also

AIH autoimmune hepatitis

HCC hepatocellular carcinoma

NASH non-alcoholic steatohepatitis

Unless otherwise indicated, data are expressed as number (percentage) of patients

months. Post-operative survival was noted. Statistical comparisons were made using the signed rank test for continuous data. Categorical data were analyzed by McNemar's test or a Wald test for marginal homogeneity. Survival data was compared using a stratified Cox proportional hazards model. $\mathrm{P}<0.05$ was considered statistically significant (Table $\mathbf{1}$ ).

\section{RESULTS}

Demographic data for both LD and DD groups are shown in Table 2. A total of 40 living donor liver transplants were carried out at our institution during the study time period. There were four gender mismatches in order to match for disease, age, and etiology. The DD group had a significantly higher median high body mass index of $26.0 \mathrm{~kg} / \mathrm{m}^{2}$ compared to $24.0 \mathrm{~kg} / \mathrm{m}^{2}$ in the LD group ( $\mathrm{p}=0.01$ ), and also a significant difference between body mass index categories $(\mathrm{p}=0.02)$. Calculated MELD scores were lower in the LD group than in the DD group (median 14 vs. 18, $\mathrm{p}=0.04$ ).

Intraoperative variables are presented in Table 3. Anesthesia time was longer in LD group with a median of 7.1 hours compared to 6.5 hours in DD $(p=0.02)$. Longer surgical time was noted for LD with median 5.4 hours compared to 5.2 hours, but this was not statistically significant $(\mathrm{p}=0.08)$. Transfusion of blood, blood product or albumin did not differ between the two groups. The frequency of intraoperative events was similar in the two

Table 3. Intraoperative Variables

\begin{tabular}{|c|c|c|c|c|c|}
\hline \multirow{2}{*}{ Variable } & \multicolumn{2}{|c|}{ Living Donor } & \multicolumn{2}{|c|}{ Deceased Donor } & \multirow{2}{*}{ p-value } \\
\hline & Median & IQR & Median & IQR & \\
\hline Surgical time, hours & 5.4 & $4.7-6.8$ & 5.2 & $4.2-6.3$ & 0.08 \\
\hline Anesthesia time, hours & 7.1 & $6.4-8.3$ & 6.5 & $5.8-7.6$ & 0.02 \\
\hline Autologous transfusion, units & 2.0 & $1.0-5.5$ & 2.0 & $1.0-6.5$ & 0.49 \\
\hline Packed red blood cells transfusion, units & 2.0 & $0-4.5$ & 2.0 & $0-5.0$ & 0.84 \\
\hline Fresh Frozen Plasma, units & 0 & $0-4.0$ & 0 & $0-6.0$ & 0.65 \\
\hline Platelets transfusion, 6 pack & 0 & $0-6.0$ & 0 & $0-6.0$ & 0.41 \\
\hline Any intraoperative event ${ }^{\dagger}, \#(\%)$ of patients & 4 & $10 \%$ & 7 & $18 \%$ & 0.55 \\
\hline
\end{tabular}

IQR: interquartile range, $25^{\text {th }}$ percentile to $75^{\text {th }}$ percentile

${ }^{\dagger}$ MAP $<50 \%$ of baseline $(\mathrm{p}=0.55)$, CPR required $(\mathrm{p}=1.00)$, hypoxemia requiring intervention $(\mathrm{p}=1.00)$, or arrhythmia requiring intervention $(\mathrm{p}=1.00)$ 
Table 4. Postoperative Complications, Number of Events (\%)

\begin{tabular}{|c|c|c|c|}
\hline Event & Living Donor & Deceased Donor & p-value \\
\hline Wound infection & $4(10 \%)$ & $3(8 \%)$ & 1.00 \\
\hline Hepatic Artery Thrombosis & $6(16 \%)$ & $1(3 \%)$ & 0.13 \\
\hline Portal Vein Thrombosis & $0(0 \%)$ & $2(5 \%)$ & 0.50 \\
\hline Biliary stenosis & $1(3 \%)$ & $0(0 \%)$ & 1.00 \\
\hline Cholestasis & $2(5 \%)$ & $0(0 \%)$ & 0.50 \\
\hline Graft nonfunction & $1(3 \%)$ & $0(0 \%)$ & 1.00 \\
\hline Sepsis & $1(3 \%)$ & $1(3 \%)$ & 1.00 \\
\hline
\end{tabular}

* Immunosuppressant toxicity, supraventricular arrhythmia, line infections, acute cellular rejection that resolved, and traumatic urinary catheter removal Note: $\mathrm{N}=39$ pairs used in comparisons, except for Hepatic artery thrombosis (HAT) $(\mathrm{N}=38)$ due to missing outcomes

groups. There was one intraoperative death in the LD group and none in the DD group.

Postoperative transfusion of fresh frozen plasma, cryoprecipitate, platelets, and albumin showed no significant differences, however, the DD group had a significantly higher postoperative packed red blood cells transfusion $(\mathrm{p}=0.04)$. Intensive care unit LOS did not significantly differ between the groups with LD median of 0.95 days compared to 0.96 days in DD. Hospital LOS was significantly longer for LD (median of 12 days ) versus DD (8 days , $\mathrm{p}=0.002$ ).
Postoperative complications are compared in Table 4. There were no significant differences in the variables.

Biliary complications (leak or stenosis) were more frequent in the LD group (5 vs. 1) and the frequency of hepatic artery thrombosis (HAT) was also greater in the LD group (6 versus 1). Neither difference reached statistical significance. One LD required relisting on postoperative day 19, with $\mathrm{HAT} / \mathrm{stenosis}$ requiring intervention. Two LD patients underwent early retransplantation (within 90 days of transplant) for HAT, and none of the DD patients required early

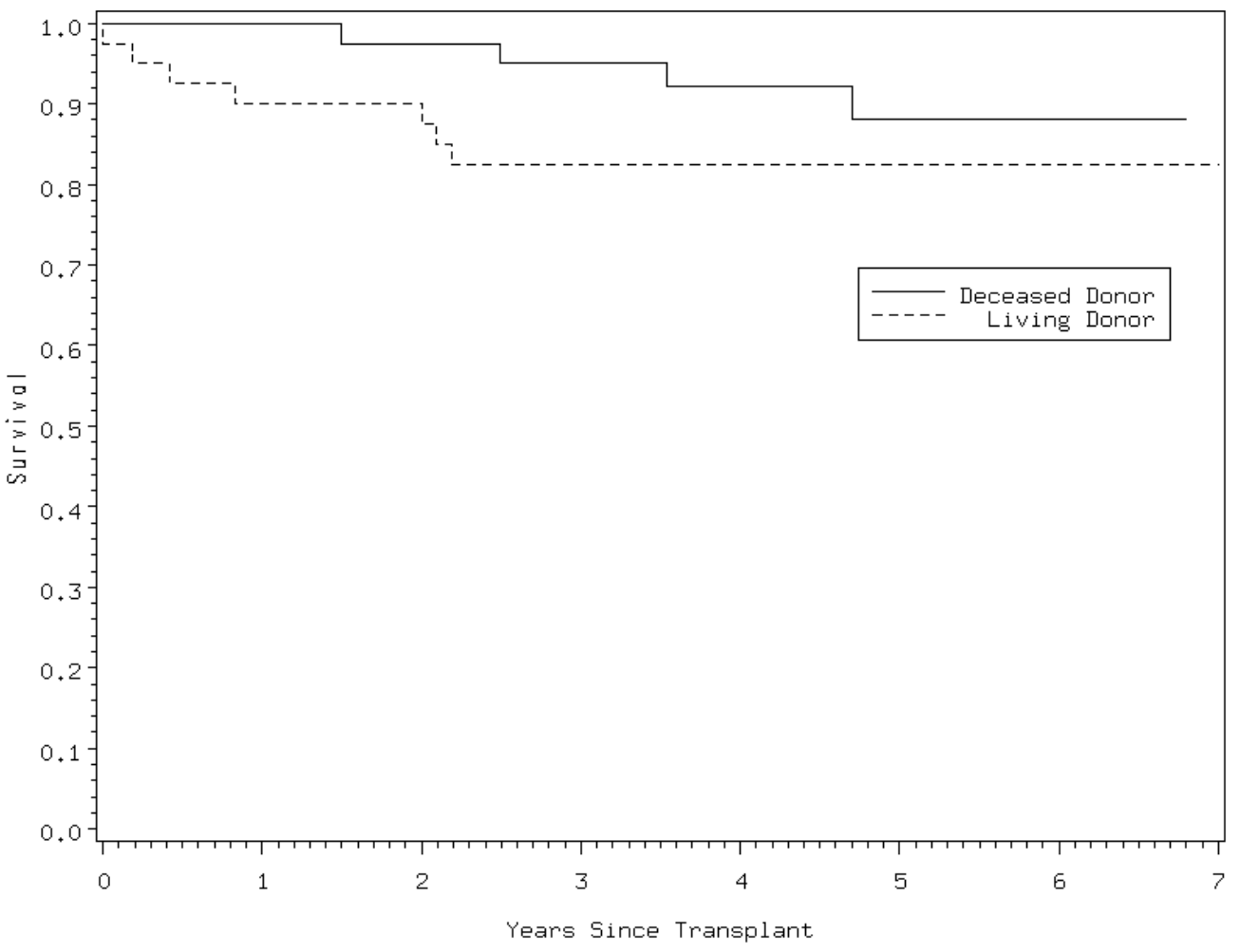

Fig. (1). Kaplan-Meier survival curves of DD and LD. $\mathrm{p}=0.118$ by stratified Cox proportional hazards model. 
retransplantation. None of the DD group were relisted in the 90 days after transplantation.

The Clavien scale was utilized to compare complications.[8,9] Chi-Square analysis comparing Clavien Grades 01 with Grades $2-4$ was not significant $(\mathrm{p}=0.254)$. Please see Table 5.

There were 7 total deaths in the LD group (6 in the postoperative period), and 4 in the DD group at the time of data collection (July 31, 2007). Fig. (1) displays the KaplanMeier survival curves. At 7 years post-transplant the estimated death rates were $18 \%$ and $12 \%$ for LD and DD, respectively. The hazard ratio comparing LD to DD was 3.50 (95\% CI 0.73-16.85, $\mathrm{p}=0.118)$.

\section{DISCUSSION}

We found that $L D$ recipients had a longer anesthesia time and longer hospital stay than matched DD recipients. There were more biliary complications and HAT in the LD group but these differences did not reach statistical significance. Otherwise postoperative morbidity was similar between LD and DD groups. This implies that the greater number of complications in LD patients resulted in longer hospital stays, however whether other intergroup differences contributed should be discussed.

The mean calculated MELD scores are low for patients undergoing liver transplantation. This is probably related to the index group being the LD recipients. More than $25 \%$ of this group underwent transplantation for tumors (cholangiocarcinoma, hepatocellular carcinoma, neuroendocrine tumor), such patients typically have low calculated MELD scores. Matching by primary etiology for transplantation will hence introduce an equal group of such patients into the DD group. The mean MELD in the DD group was significantly higher than in the LD group. This would imply longer hospital stay in this group likely due to postoperative complications $[10,11]$ rather than the shorter time observed.

The DD group had a significantly higher BMI which may also increase the complication rate, although previous reports of the influence of obesity on post-transplant complications are not uniform [12-15]. Again, this would tend to result in a longer hospital stay in the DD group, contrary to our findings.

It is also possible that the longer hospital stay in the LD group was a result of a more conservative approach to hospital discharge in these patients than in the DD group. We have no way of assessing this possibility with the data available. A recent study from the Adult-to-Adult Living Donor Liver Consortium (A2ALL) reported a median LOS of 13 days in LD recipients, similar to our LD group [4].

There was a longer total operating room time in the LD group (median 7.1 hours compared to 6.5 hours), despite little difference in the surgical times. Both patient groups received the same invasive monitors and line placement, although some DD came to the operating room from the ICU, and may have some lines in place prior to anesthetic induction. In addition one anesthesiologist supervised both the donor and recipient cases, which may have contributed to the longer operating room time. Another factor may be our attempts to appropriately coordinate the donor and recipient procedures, which may have resulted in longer time between induction of anesthesia and incision in the LD group. Our median surgical time for LD was 5.4 hours, which compares favorably with recently reported operative times with a mean of 8.5 hours and median of 11.9 hours $[3,4]$.

\section{ADDENDUM 1}

Table 5. Graded Complications of Living Donor and Deceased Donor Recipients

\begin{tabular}{|c|c|c|c|}
\hline Clavien Grade & Clavien Definition & LD & DD \\
\hline 1 & $\begin{array}{l}\text { Any deviation from the normal postoperative course without the need for pharmacological treatment or surgical, endoscopic, and } \\
\text { radiological interventions. Allowed therapeutic regimens are: drugs as antiemetics, antipyretics, analgesics, diuretics, electrolytes, } \\
\text { and physiotherapy. This grade also includes wound infections opened at the bedside. }\end{array}$ & 10 & 18 \\
\hline 2 & $\begin{array}{l}\text { Requiring pharmacological treatment with drugs other than such allowed for grade I complications. Blood transfusions and total } \\
\text { parenteral nutrition are also included. }\end{array}$ & 16 & 13 \\
\hline 3 & $\begin{array}{l}\text { Requiring surgical, endoscopic or radiological intervention } \\
\text { a. Intervention not under general anesthesia } \\
\text { b. Intervention under general anesthesia }\end{array}$ & \begin{tabular}{c|c}
6 \\
11
\end{tabular} & $\begin{array}{l}8 \\
6\end{array}$ \\
\hline 4 & $\begin{array}{l}\text { Life-threatening complication (including central nervous system complications)* requiring intermediate care/intensive care unit } \\
\text { management } \\
\quad \text { a. Single organ dysfunction (including dialysis) } \\
\quad \text { b. Multi-organ dysfunction }\end{array}$ & $\begin{array}{l}1 \\
0\end{array}$ & $\begin{array}{l}1 \\
0\end{array}$ \\
\hline 5 & Death of a patient & 0 & 0 \\
\hline
\end{tabular}


Comparison of overall intraoperative event rates was not significantly different between the LD and DD groups. We observed no difference in transfusions of blood or blood products. Our transfusion amounts for packed red blood cells in the LD group was slightly lower than those reported in the Adult-to-Adult Living Donor Liver Transplantation Cohort Study (A2ALL) for LD [4]. We had $75 \%$ of LD receive $0-4$ units packed red blood cells, compared to $47 \%$ in the A2ALL study [4]. In the A2ALL results, $14 \%$ of LD received $13-56$ units, $15 \%$ received $9-12$ units, and $24 \%$ received 5-8 units of packed red blood cells. The one intraoperative death in the LD group was due to uncontrollable hemorrhage in a patient with diffuse intrahepatic neurovascular cancer and multiple extensive prior upper abdominal operations.

There were no significant differences in any of the postoperative complications we assessed; however biliary complications and HAT were more frequent in the LD group. Biliary complications have recently been reported in $12.9 \%$ of LD at 1 year post-transplant, which is similar to our $15 \%$ in our initial LD experience [16]. In other recent publications, incidence of $30-33 \%$ biliary leaks and $8-24 \%$ biliary stricture are noted in LD $[3,4]$. Our data did show a trend towards more biliary complications in LD, and was significantly increased in the A2ALL Cohort Study [6].

Wound infections were noted in $10 \%$ of $\mathrm{LD}$ and $7.7 \%$ of DD. Wound infections were previously reported in $37 \%$ of LD performed in 2001 and 2002 by Iinuma et al., and 32\% in the A2ALL study [4,17].

Acute renal dysfunction developed in 5\% of LD compared to $10 \%$ of DD, which was not a significant difference. We defined acute renal dysfunction as change from baseline creatinine of greater than $50 \%$. Recently, Akamatsu et al. reported a 29\% incidence of renal impairment, with a definition of this as serum creatinine of greater than 1.5 in patients with normal baseline creatinine [18]. The difference between our findings and those from Akamatsu may be due to variation in definition.

Complications by Clavien classification did not reveal significant differences between the groups, with comparison of Grades 0-1 with Grades 2-5. Our assessment of complications in Table 4 included rehospitalization, which was counted in addition to reason for rehospitalization in the first three months after transplant. This was not done in the Clavien grading. There appeared to be more grade $3 \mathrm{a}$ and $3 \mathrm{~b}$ complications in the LD group, but this did not reach statistical significance. Friese et al. found higher early complications (with first $20 \mathrm{LD}$ ) performed at a center, but this decreased with more experience [6]. Our finding of no difference between LD and DD with our first 40 cases may be attributable to single center $v s$. multiple center experience.

There were 4 deaths in our LD within 1 year of transplant, which corresponds to a $90 \%$ one-year survival from a Kaplan-Meier survival curve (Fig. 1). This is comparable to published data - recently Morioka reported a $73 \% 1$ year, Ghobrial et al report an $88 \% 1$ year, and the A2ALL study reported a $89 \% 1$ year survival $[3,4,19]$. Causes of death in the LD group included metastatic disease (cholangiocarcinoma) and multi-system organ failure. These were also seen the in DD group, in addition to newly diagnosed cancer. Comparison of death rates at the time of last follow-up was not statistically significant. The hazard ratio for LD was 3.50 but the wide $95 \%$ confidence interval (0.73-16.85) warns against reading any significance into this value.

Our study has limitations. It is retrospective. The statistical power is limited both by the number of patients ( 40 cases, 40 controls) and the small number of events in the study groups. Because of this we cannot determine if our finding of a non-statistically significant difference in complication between LD and DD groups reflects a true difference as in previous studies $[3,4,19]$, or whether in our series the rates do not differ significantly. Altering the case:control matching, for example to $1: 2$ rather than $1: 1$ could potentially increase the power; however, we were obliged to extend our age range for matching (from 10 to 12 years) and accept 4 gender mismatches to conclude a 1:1 ratio so this was not feasible. To resolve this issue a reanalysis of our data after more LD have been performed will be necessary.

\section{CONCLUSION}

This case controlled study comparing LD to DD recipients demonstrated longer OR time and longer hospital stays for LD recipients. There was tendency toward more biliary and vascular complications in the LD recipients which may account for the longer hospital stays. Patient survival was similar. In order to optimize our understanding of perioperative outcomes in LD recipients versus DD recipients, a larger, prospective, multi-center, study would be necessary. For now practitioners should be aware of the potential for postoperative differences between the two groups based on both retrospective single-center and multicenter studies.

\section{ACKNOWLEDGEMENTS}

Drs. Gali, Plevak, Nagorney, Rosen and Findlay has designed the research work, study and has practically performed the research work. Data analysis done by Drs. Gali, Plevak, Rosen, Dierkhising and Findlay. Data collection has been done by Drs. Gali, Plevak, Rosen and Findlay. Paper has written by Drs. Gali, Plevak, Nagorney, Rosen, Heimbach, Dierkhising and Findlay.

\section{FUNDING SOURCES}

Support was provided solely from institutional and/or departmental sources.

\section{REFERENCES}

[1] 2007 OPTN/SRTR Annual Report 1997-2006. HHS/HRSA/ HSB/DOT. [cited 06/10/2008]. Available from: http://www optn.org/AR2007/

[2] Ghobrial RM, Saab S, Lassman C, et al. Donor and recipient outcomes in right lobe adult living donor liver transplantation. Liver Transpl 2002; 8: 901-9.

[3] Morioka D, Egawa H, Kasahara M, et al. Outcomes of adult-toadult living donor liver transplantation: a single institution's experience with 335 consecutive cases. Ann Surg 2007; 245: 31525

[4] Olthoff KM, Merion RM, Ghobrial RM, et al. Outcomes of 385 adult-to-adult living donor liver transplant recipients: a report from the A2ALL Consortium. Ann Surg 2005; 242: 314-23. 
[5] Shiffman ML, Brown RS, Jr., Olthoff KM, et al. Living donor liver transplantation: summary of a conference at The National Institutes of Health. Liver Transpl 2002; 8: 174-88.

[6] Freise CE, Gillespie BW, Koffron AJ, et al. Recipient morbidity after living and deceased donor liver transplantation: findings from the A2ALL Retrospective Cohort Study. Am J Transplant 2008; 8: 2569-79.

[7] Mantel HT, Rosen CB, Heimbach JK, et al. Vascular complications after orthotopic liver transplantation after neoadjuvant therapy for hilar cholangiocarcinoma. Liver Transpl 2007; 13: 1372-81.

[8] Clavien PA, Camargo CA, Jr., Croxford R, et al. Definition and classification of negative outcomes in solid organ transplantation. Application in liver transplantation. Ann Surg 1994; 220: 109-20.

[9] Dindo D, Demartines N, Clavien PA. Classification of surgical complications: a new proposal with evaluation in a cohort of 6336 patients and results of a survey. Ann Surg 2004; 240: 205-13.

[10] Silberhumer GR, Pokorny H, Hetz H, et al. Combination of extended donor criteria and changes in the Model for End-Stage Liver Disease score predict patient survival and primary dysfunction in liver transplantation: a retrospective analysis. Transplantation 2007; 83: 588-92.

[11] Washburn WK, Pollock BH, Nichols L, Speeg KV, Halff G. Impact of recipient MELD score on resource utilization. Am J Transplant 2006; 6: 2449-54.
[12] Braunfeld MY, Chan S, Pregler J, et al. Liver transplantation in the morbidly obese. J Clin Anesth 1996; 8: 585-90.

[13] Hillingso JG, Wettergren A, Hyoudo M, Kirkegaard P. Obesity increases mortality in liver transplantation--the Danish experience. Transpl Int 2005; 18: 1231-5.

[14] Nair S, Verma S, Thuluvath PJ. Obesity and its effect on survival in patients undergoing orthotopic liver transplantation in the United States. Hepatology 2002; 35: 105-9.

[15] Sawyer RG, Pelletier SJ, Pruett TL. Increased early morbidity and mortality with acceptable long-term function in severely obese patients undergoing liver transplantation. Clin Transplant 1999; 13 126-30.

[16] Hwang S, Lee SG, Sung KB, et al. Long-term incidence, risk factors, and management of biliary complications after adult living donor liver transplantation. Liver Transpl 2006; 12: 831-8.

[17] Iinuma Y, Senda K, Fujihara N, et al. Surgical site infection in living-donor liver transplant recipients: a prospective study. Transplantation 2004; 78: 704-9.

[18] Akamatsu N, Sugawara Y, Tamura S, et al. Renal impairment after living donor liver transplantation. Transplant Proc 2006; 38: 14746.

[19] Ghobrial RM, Busuttil RW. Challenges of adult living-donor liver transplantation. J Hepatobiliary Pancreat Surg 2006; 13: 139-45.

(c) Gali et al.; Licensee Bentham Open.

This is an open access article licensed under the terms of the Creative Commons Attribution Non-Commercial License (http://creativecommons.org/ licenses/by-nc/3.0/), which permits unrestricted, non-commercial use, distribution and reproduction in any medium, provided the work is properly cited. 